\title{
Invariant Moments Based Feature Extraction for Handwritten Devanagari Vowels Recognition
}

\author{
R. J. Ramteke \\ Department of Computer Science \\ North Maharashtra University, \\ Jalgaon, (Maharashtra), \\ INDIA 425001.
}

\begin{abstract}
In this paper, a system based Handwritten Devanagari Character Recognition (HDCR) is proposed. The paper presents an experimental assessment of the efficiency of various methods based on Invariant Moments for handwritten devanagari vowels recognition. The technique is independent of size, slant, orientation, translation and other variations in handwritten vowels. For segmentation of the devanagari words, the header line (Shirorekha), plays vital role. The same tool with vertical and horizontal projection has been adapted to isolate the 13 vowels in five different groups. In order to enhance the performance of the system, an attempt has been made to compute invariant moments by small perturbation in image and information is extracted from the perturbation. But it was found that, another local feature descriptor, image partition in different zoning is better representation of the features than perturbation. The other method of image partition with different ways found better. 10 samples of each vowel from 25 people have been sampled and a database was prepared. Individual image is normalized to $40 \mathrm{X} 40$ pixel size. The Fuzzy Gaussian Membership function has been adopted for classification. The success rate of the method is found to be 94.56 .
\end{abstract}

\section{Categories and Subject Descriptors}

I.5.2 [Pattern Recognition]: Design Methodologies - classifier design and evaluation, feature evaluation and selection, pattern analysis.

\section{General Terms}

Algorithms, Measurement, Documentation, Performance, Design, Experimentation, Human Factors, Languages, Verification.

\section{Keywords}

Invariant Moments, Handwritten Devanagari Vowels Recognition, OCR, Segmentation, Fuzzy Membership Function, Image perturbation.

\section{INTRODUCTION}

WRITING, which has been the most natural mode of collecting, storing, and transmitting information through the centuries, now serves not only for communication among human but also serves for communication of humans and machines. Handwriting recognition is the task of transforming a language represented in

its spatial form of graphical marks into its symbolic representation, typically the ASCII representation of characters.
The intensive research effort in the field of character recognition was not only because of its challenge on simulation of human reading but also because it provides efficient application such as the automatic processing of bulk amount of papers, transferring data into machines, and web interface to paper documents.

Character recognition can be classified as: on-line or off-line, printed or handwritten, constrained or unconstrained. No Matter in which class the problem belongs. In general, there are five major stages in CR problem: Image pre-processing, segmentation, feature extraction, Training \& Recognition and Post processing.

Most of the Indian scripts are distinguished by the presence of the matras in addition to main characters as against the English script that has no matras. Therefore, algorithms developed for them are not directly applicable to Indian scripts. Many OCRs for Indian scripts have been reported $[1,2,3,4]$. However, very few of these have attempted the handwritten Devanagari character. Printed Devanagari character recognition is attempted based on Kohenen Neural Network (KNN) and Neural Network [4,16]. These results are extended to Bangla [5], which also has the header line like Devanagari. Structural features like concavities and inter-sections are used as features. A similar approach is tried for Gujrati [6] with limited success. Reasonable results are reported for Gurumukhi Script [7]. Two-Stage classification and feature extraction technique is used for Arabic handwritten digit recognition [8]. Unlike English and other Roman scripts, Devanagari has a few, if any, commercial OCR renders; and the ones that have products provide only the custom enterprise solutions. The basic components of the system however are described in the literature [5]. After word and character segmentation, a feature based tree classifier is used to recognize the basic characters. Error detection and correction for the OCR based on the dictionary search has led to the recognition accuracy of $91.25 \%$ at the word level and $97.18 \%$ at the character level on clean images.

Bansal [9] has designed a Devanagari text recognition system by integrating knowledge sources, features of the characters such as horizontal zero crossing, moments, aspect ratios, pixel density in nine zones, number, and positions of vertex points, with structural descriptions of characters. These are used to classify characters and perform recognition. On printed Devanagari recognition rates of approximately $70 \%$ without any post-processing and $88 \%$ correct recognition with the help of a word dictionary are reported. It should be noted that both of the above OCR system need vast amount of training data to achieve an acceptable level of performance. 
In character recognition problem, the description phase plays a fundamental role, since it defines the set of properties, which are considered essential for characterizing the pattern. Moments \& function of moments have been utilized as pattern feature in number of application [10]. Using non-linear combinations of geometric moments, a set of invariant moments has been derived, which has the desirable properties of being invariant under image translation, scaling, rotation \& reflection. A number of papers describing application of invariant moment [11] with its types (e.g. complex moments, rotational moments, Zernike moments, Legendre moments etc) have been published.

In the field of character recognition, it is now established that a single feature extraction method and a single classification algorithm generally cannot yields a very low error rate. Therefore it is proposed that the combination of features can create better success rates. Three major interest can however justify such an approach (i) the use of several types of features still ensures an accurate description of the characters; (ii) the use of a single classifier preserves fast and flexible learning; (iii) the tedious tuning of combination rule is avoided.

In the present work a standardized database has been created first with respect to variety in handwriting style. The results reported in the paper are more reliable and satisfactory as compared to existing techniques in terms of features, classifiers and environment. The paper is organized as follows. Section 2 deals with introduction to Devanagari Script and vowels. In section 3, the segmentation of within vowels to obtain core character is presented. The fundamental theorem of Invariant Moments is presented in section 4. The theory of different methods experimented is discussed in section 5. Section 6 gives details of Gaussian distribution method for recognition. The section 7 provides discussion regarding results and conclusion is summarized in section 8 .

\section{DEVANAGARI SCRIPT AND VOWELS}

India is multilingual country of more than 1 billion population with 18 constitutional languages and 10 different scripts. Devanagari, an alphabetic script, is used by a number of Indian Languages. It is believed that Brahmins invented Devanagari script in order to conceal knowledge from the common person. Non-Brahmins were not able to learn it due to the difficult nature of the script, which enabled the Brahmins to maintain their stranglehold over the non-Brahmin races and crush them into a sub-human existence [12]. It was adopted by many other languages such as Marathi, Hindi, Konkani, and Nepali. Many other Indian languages use close variant of this script. Hindi is world's third most commonly used language after English and Chinese, and there are approximately 500 million people all over the world that speak and write in Hindi.

Devanagari has about 13 vowels (Shown in figure 1) and 34 consonants. In addition, there are large number of conjuncts formed by combination of consonants, their half-forms and some modifier symbols. The modifier symbols are placed either on the top, at the bottom, on the left, to the right or a combination of these. A horizontal line called Shirorekha (a header line) runs through the entire span of word. Top modifiers are placed above the header line. The lower modifiers are placed below the character.

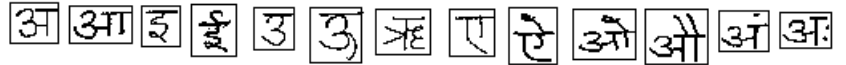

Figure 1. Handwritten Devanagari Vowels

As no standardized database for Devanagari Handwritten Characters is available, the database has been created. A special sheet is designed for data collection. Data is collected from people domain with 10 samples of each character from about 25 persons from different fields and age. Data acquisition is done manually. The noise removal process has been done at the same time.

\section{SEGEMENTATION AND CLUSTERING}

Segmentation process involves separation of a word into characters, lower modifiers, upper modifiers and separation of conjunct characters into consonants and half consonants. Segmentation of handwritten Devanagari text involves the following steps: (i) scanning of text, (ii) Lines and words segmentation (iii) Binarization of word images (iv)Thinning of segmented binary word images and (v) Segmentation of words into characters and modifiers. In this work, we use a projection approach [13] for segmentation of handwritten vowels to obtain core devanagari vowel, which is further clustered in the group of similar appearance vowels. For examples, following are the clusters of similar look.

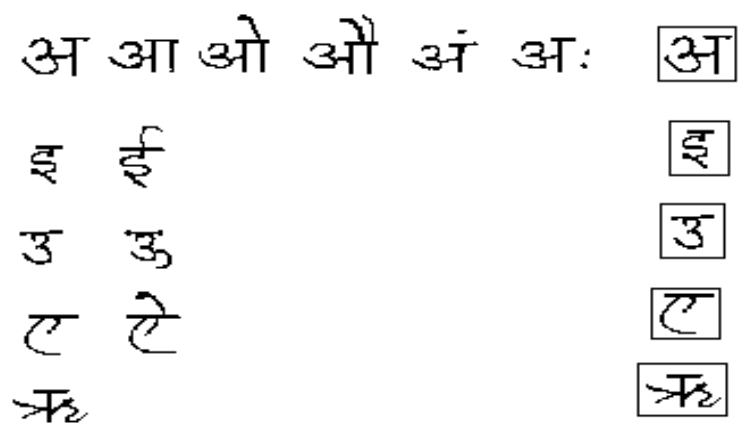

Figure 2: Five cluster of the vowels of similar appearance

To achieve the said clusters, devanagari script has a powerful tool called Shirorekha, header line. It is the most visible and distinguishing part of a character or word. By separating the header line, we can obtain the top and core-bottom part of a character. For separation of header line, the horizontal density (i.e. the number of pixel in each row) of the vowel is calculated and the region with maximum density lying within the top 3/4th of the word is identified. The top 3/4th of a vowel has been considered because the upper modifiers lie within the top half of the word. Taking the top 3/4th area will allow us account for such samples. Since the header line covers the entire word, the region with the highest pixel density will give the position of the line. In the handwritten characters the header line covers multiple rows in contrast to printed characters whereas it covers a single row. Once the position of the header line is determined, it has been removed. Further, matra is removed by taking vertical projection of the character, where there is a gap between two columns, the matra is segmented. As a result, the core character was achieved. Figure 3 shows the segmentation of conjunct vowel in core vowel. 


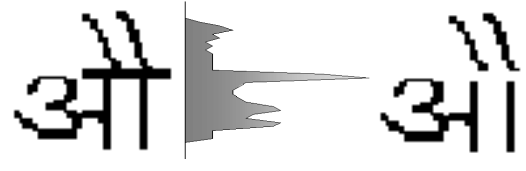

(a)

(b)

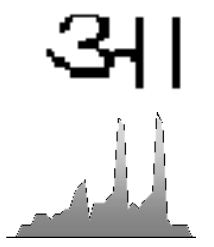

अ

(d)

( c)

Figure 3. (a) Original vowel image with its horizontal projection (b)Without header line vowel image (c) Vertical projection of vowel image without top modifier (d) A core vowel.

\section{FEATURE EXTRACTION}

To divide each group, we use the features drawn by invariants moment technique which is used to evaluate seven distributed parameter of a character image. The moment invariants (IMs) are well known to be invariant under translation, scaling, rotation and reflection $[10,11,14]$. They are measures of the pixel distribution around the centre of gravity of the character and allow to capture the global character shape information. In the present work, the moment invariants are evaluated using central moments of the image function $f(x, y)$ ) up to third order.

Regular moments are defined as

$\mu_{p q}=\sum_{X} \sum_{Y}(X-\bar{X})^{p}(Y-\bar{Y})^{q} f(X, Y)$

where for $\mathrm{p}, \mathrm{q}=0,1,2, \ldots$ and $\bar{X}$ and $\bar{Y}$ are moments evaluated from the geometrical moments $m_{\mathrm{pq}}$ as follows,

$$
\begin{aligned}
& \bar{X}=m_{10} / m_{00} \text { and } \bar{Y}=m_{01} / m_{00} \\
& m_{p q}=\sum_{X} \sum_{Y} X^{p}{ }^{q} f(X, Y)
\end{aligned}
$$

The normalized central moment to shape and size of order $(p+q)$ is defined as

$$
\eta_{p q}=\mu_{p q} / \mu_{00}^{\gamma}
$$

for $\mathrm{p}, \mathrm{q}=0,1,2, \ldots$ Where $\quad \gamma=\frac{(p+q)}{2}+1$

for $(p+q)=2,3, \ldots$

A set of seven moment invariants can be derived from equations (6)

In this work each segmented character is resized as $40 \mathrm{X} 40$ pixel image. The image matrix $f(x, y)$ is processed to obtain the character with white color on black background. The expressions given by Equations (6) are used to evaluate 7 central invariants moment i.e. $\phi_{1}=\eta_{20}+\eta_{02}$

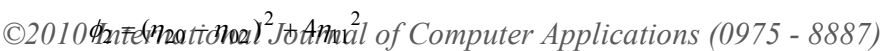

$$
\begin{aligned}
\phi_{3}= & \left(\eta_{30}-3 \eta_{12}\right)^{2}+\left(3 \eta_{21}-\eta_{03}\right)^{2} \quad \text { Volume } \\
\phi_{4}= & \left(\eta_{30}+\eta_{12}\right)^{2}+\left(\eta_{21}+\eta_{03}\right)^{2} \\
\phi_{5}= & \left(\eta_{30}-3 \eta_{12}\right)\left(\eta_{30}+\eta_{12}\right)\left[\left(\eta_{30}+\eta_{12}\right)^{2}-3\left(\eta_{21}+\eta_{03}\right)^{2}\right] \\
& +\left(3 \eta_{21}-\eta_{03}\right)\left(\eta_{21}+\eta_{03}\right)\left[3\left(\eta_{30}+\eta_{12}\right)^{2}-\left(\eta_{21}+\eta_{03}\right)^{2}\right] \\
\phi_{6}= & \left(\eta_{20}-\eta_{02}\right)\left[\left(\eta_{30}+\eta_{12}\right)^{2}-\left(\eta_{21}+\eta_{03}\right)^{2}\right]+ \\
& 4 \eta_{11}\left(\eta_{30}+\eta_{12}\right)\left(\eta_{21}+\eta_{03}\right) \\
\phi_{7}= & \left(3 \eta_{21}-\eta_{03}\right)\left(\eta_{30}+\eta_{12}\right)\left[\left(\eta_{30}+\eta_{12}\right)^{2}-3\left(\eta_{21}+\eta_{03}\right)^{2}\right] \\
& +\left(3 \eta_{12}-\eta_{30}\right)\left(\eta_{21}+\eta_{03}\right)\left[3\left(\eta_{30}+\eta_{12}\right)^{2}-\left(\eta_{21}+\eta_{03}\right)^{2}\right]
\end{aligned}
$$

$\left(\Phi_{1}-\Phi_{7}\right)$ which are used as features. In experiment, the invariant moments are evaluated by taking log of the absolute value of the moment of each of the sample image of the numerals [15]. Further, mean and standard deviation are determined for each feature using samples. Thus we had 14 features $(7$ means and 7 standard deviations). Fuzzy Gaussian Membership function has been adopted for classification. To increase the success rate, the new features need to be extracted based on perturbation and divisions of the images.

\subsection{Perturbed Moments}

The IMs, $\Phi^{\prime}$ s, are invariant under scaling, translation and rotation operation. The success depends upon its sensitivity with respect to distribution of pixel for a given character set. Therefore, it is useful if we analyse the sensitivity analysis of these invariant moments with respect to pixel distribution.

If an image $f(x, y)$ gets disturbed by small amount to $f\left(x^{\prime} y^{\prime}\right)$, where the $(\mathrm{x}, \mathrm{y})$ point is shifted to new point $\left(\mathrm{x}^{\prime}, \mathrm{y}^{\prime}\right)$, the invariant moments also get changed to new values as $\Phi_{i}^{\prime}$ s. Since invariant moments are not invariant under shearing operation, it is useful to perturbed the image by shearing operation, as

$$
\left(\begin{array}{ll}
X & Y
\end{array}\right)\left(\begin{array}{ll}
1 & a \\
b & 1
\end{array}\right)=\left(\begin{array}{ll}
X+b Y & Y+a X
\end{array}\right)
$$

Where $\mathrm{a}$ and $\mathrm{b}$ are perturbation parameters. In our work, we have chosen $\mathrm{a}=\mathrm{b}=0.1$. Therefore equation (2) can be written as

$$
M_{p q}^{\prime}=\sum_{X} \sum_{Y}(X+b Y)^{p}(Y+a X)^{q} f(X, Y)
$$

and new perturbed differential moments can be defined as

$$
\Delta \phi^{\prime}(a, b)=\frac{\left(\phi^{\prime}(a, b)-\phi\right)}{\phi}
$$

The change is combined with IMs and applied for the recognition. The success rate was not in the promising stage, therefore the new features need to be extracted based on and divisions of the images. These new features are useful to improve the recognition rate.

\subsection{Image Partition}

The equations in section 4 are used for the extraction of feature of each character. As the property of invariant moment discussed above i.e. invariant under reflection, there is problem in recognition because of similarity under reflection. The recognition rate was found to be only $49.2 \%$ by using the seven invariants of each numeral. Therefore, the image is divided into 4 zones with three feature sets on the basis of centroid of the character image $(\bar{X}, \bar{Y})$

\subsubsection{Feature set 1}

After getting the centroid, we evaluated the invariant moments features of each parts. Thus in total there are 28 features. All these 
features are used in the recognition system. By including these 28 features the recognition rate can be enhanced to $74.82 \%$ from $39 \%$. A devanagari handwritten vowel ' $अ$ ' and its division is shown in fig. 3.

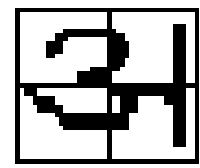

Fig. 3 vowel 'अ' (A) Divided into 4 Parts

\subsubsection{Feature Set 2}

Another way of extracting features from image is dividing image into two zones. This method gives more information with different features as compared to feature set 1 . Four zones (2 vertical \& 2 horizontal ) of an image is shown in figure 4 .

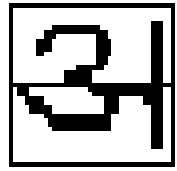

Fig. $4(A)$

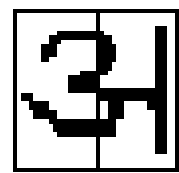

(B)
Fig. 4 (A) vowel 'अ'

(A) Divided vertically into 2 zones ( Up and Down)

(B) Divided horizontally into 2 zones (Left and Right)

The MIs features of each parts i.e. Left, Right, Up and down are evaluated. Thus 28 more features (7 of each parts) are extracted. Further we tried these 28 features separately with original 7 features (i.e. total 35 features) for recognition. The success rate is found to be less than the rate corresponding to feature set 1 .

\subsubsection{Feature Set 3}

The diagonal zones can also be used for specific information. The 4 parts of Fig 3, can be arranged in such a fashion so that the diagonal parts can be used as another feature set. Left diagonal and right diagonal image has 14 more features. After adding all the feature sets with original features the recognition rate is found to be about $92 \%$. This is significant increase in success rate.
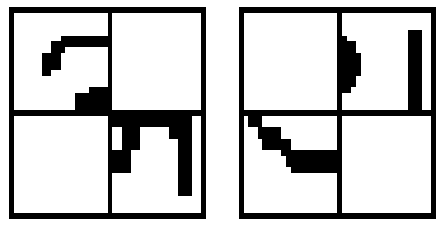

Fig. 5 (A) Right diagonal

(B) Left diagonal

\section{RECOGNITION SYSTEM}

A database of vowels has been required to extract the features, which form a template (Trained Database). The mean and standard deviation are computed for each type of features corresponding to each value of the characters. The unknown character features are matched with all reference character features available in the each group and found the maximum membership value $(0-1)$ of the character with the reference character in the corresponding group. The template consists of mean $\mathrm{Mi}$ and standard deviation $\sigma$ i for each feature and computed as [3]:

Mean $\quad M_{i}=\frac{1}{N_{i}} \sum \phi_{i(k)}$

Std-Dev $\sigma_{i}=\sqrt{\sum\left(\phi_{i(k)}-M_{i}\right)^{2}}$

Where $\mathrm{N}_{\mathrm{i}}$ is the number of samples in $\mathrm{i}^{\text {th }}$ class and $\phi_{i(k)}$ stands for the $\mathrm{k}^{\text {th }}$ feature value of reference character in the $\mathrm{i}^{\text {th }}$ class.

For an unknown input numeral $\mathrm{X}$, the corresponding features are extracted. The Fuzzy Gaussian Membership Function is used to get the maximum membership value as follows:

$\mu_{X i}=\exp -\frac{\left(X_{i}-M_{i}\right)^{2}}{2 \sigma_{i}^{2}}$

where $x_{i}$ is the $i^{\text {th }}$ feature of the unknown character.

Let $\mathrm{M}_{\mathrm{j}}(\mathrm{r}), \sigma_{\mathrm{j}}^{2}(\mathrm{r})$ belongs to the $\mathrm{r}^{\text {th }}$ reference character which is any character class in the group. We then calculate the average membership value as

$\mu_{a v}(r)=\frac{1}{C} \sum_{j=1}^{C} \exp -\frac{\left(X_{j}-M_{j}\right)^{2}}{2 \sigma_{j}^{2}}$

where $\mathrm{x} \in \mathrm{r}$ if $\mu_{\mathrm{av}}(\mathrm{r})$ is the maximum for $\mathrm{r}$. An array is formed of the corresponding classes. The resultant array is then normalized in order to enhance the success rate by the following expressions.

$d_{i}(t)=\frac{1}{N} \sum_{1}^{N} d_{i}$

Where $\mathrm{N}$ is the number of classes in the group, $\mathrm{d}_{\mathrm{i}}$ is $i^{\text {th }}$ feature of class d.

Normalization $n=\left(\frac{d_{i}}{d_{i}(t)}\right) * 100$

\section{RESULT AND CONCLUSION}

13 vowels were taken into consideration for testing but as segmentation derived five major classes. Therefore, the system was tested on 5 handwritten devanagari vowels viz, अ, इ, $3, \succeq, \mp$. Overall 250 samples of each vowel were collected from people domain. Following table I shows the success rate.

Table 1. Recognition Result of vowels

\begin{tabular}{|c|c|c|c|c|}
\hline Vowels & Total Images & IMs & IMs+PM & IMs+S1+S2+S3 \\
\hline अ & 250 & 148 & 156 & 244 \\
\hline § & 250 & 103 & 144 & 229 \\
\hline З & 250 & 133 & 208 & 235 \\
\hline $\boldsymbol{Z}$ & 250 & 118 & 200 & 233 \\
\hline
\end{tabular}




\begin{tabular}{|c|c|c|c|c|}
\cline { 2 - 3 } 哽 & 250 & 113 & 156 & 241 \\
\hline & & $\mathbf{4 9 . 2 0}$ & $\mathbf{6 9 . 8 4}$ & $\mathbf{9 4 . 5 6}$ \\
\hline
\end{tabular}

IMs- Invariant Moments, PM- Perturbed Moments, S- Feature Set i

As better recognition rate was found with three sets of image partition and invariant moments features. The promising performance of $94.56 \%$ at handwritten devanagari vowel has been achieved. The clustering results has not mentioned here but are also notable and the work is in progress it will be shown in next version of the paper. Further the same technique is applying on other devanagari consonant.

Main advantage of our approach is that the features are invariants under translation, scale, rotation, and reflection. It overcomes the problems in varieties in handwriting style. Some times the handwritten characters are tilt, small or large in shape. The invariants moment features are near about same for these variations. But reflection feature creates complexity with mirror images of the vowels like $\equiv, \overline{3}$ because of their similar look.

The key to recognition system is how to divide a character. We found the solution in form of image partition for the reflection problem as they help in enhancement of success rate in spite of great variation in character due to different styles of handwriting.

\section{REFERENCES}

[1] Nafiz Arica, Fatos T. Yarman-Vural, "An Overview of Character Recognition Focused on Off-line Handwriting", IEEE Trans. on System, Man, And Cybernetics - Part C: Application and Reviews, Vol.31 No.2, pp. 216-233, May 2001.

[2] Veena Bansal, R.M.K.Sinha, "Integrating Knowledge Sources in Devanagari Text Recognition System", IEEE Trans. On System, Man and Cybernetics, Vol. 30, 4, 2000.

[3] R. J. Ramteke, V. D. Bhagile, S. C. Mehrotra, 2008, Recognition of Offline Handwritten Devanagari Characters, in Advances in Computer Vision and Information Technology, published by I. K. International Publishing House, New Delhi, ISBN- 978-81-89866-74-7, pages-12911301, 2008.

[4] Swapnil Khedekar, V.Ramanaprasad, S.Setlur, 2003, TextImage Separation in Devanagari Documents, 7th Int. Conf. on Document Analysis and Recognition (ICDAR'03), 2003.

[5] B. B. Chaudhari, U. Pal, 1997, An OCR System to read two Indian Language: Bangla and Devanagari (Hindi), 4th Int. Conference on Document Analysis and Recognition, (ICDAR'97), pp. 1011-1016, 1997.

[6] Sameer Antani, Lalita Agnihotri, 1999, "Gujarati Character Recognition", 5th Int. Conf. on Document Analysis and Recognition (ICDAR'99) 1999.

[7] G. S. Lehal, C. Singh, R. Lehal, "A shape Based Post Processor for Gurumukhi OCR", 6th Int. Conf. on Document Analysis and Recognition (ICDAR 2001), Seattle, 2001.

[8] Sherif Abdleazeem, Ezzat El-Sherif. 2008. Arabic Handwritten Digit Recognition, Internatinal Journal of
Document Analysis and Recognition (IJDAR), Vol.11, 2008, 127-141, DOI 10.1007/s10032-008-0073-5

[9] V. Bansal, R.M.K. Sinha, 2001, A Devanagari OCR nad a brief review of OCR research for Indian scripts, Proc. of STRANS01, IIT Kanpur, India 2001.

[10] R. J. Ramteke, S. C. Mehrotra, 2006. Feature Extraction Based on Invariant Moments for Handwriting Recognition, at Proc. of 2006 IEEE International Conference on Cybernetics and Intelligent System (CIS-2006), Bangkok, Thailand, ISBN: 1-4244-0023-6, DOI: $10.1109 /$ ICCIS.2006.252262, pp. $1-6$, on $7^{\text {th }}-9^{\text {th }}$ June 2006.

[11] Jan Flusser. 2000. On the independence of rotation moment invariants, Pattern Recognition 33 (2000) 1405-1410.

[12] Santosh K.C. and Cholwich Nattee, 2007. Template-based Nepali Natural Handwritten Alphanumeric Character Recognition, Thammasat Int. J. Sc. Tech., Vol. 12, No. 1, January-March 2007

[13] R. J. Ramteke, S. C. Mehrotra, 2006. Segmentation and Clustering of Handwritten Devanagari Text, Proc. of $1^{\text {st }}$ International Conference on Signal and Image Processing (ICSIP - 2006), Hubli, Karnataka, India, ISBN: 10:023063003-0, pp. 549-554, Dec. 2006.

[14] Khalid M. Hosny. 2007, Efficient Computation of Legendre Moments for Gray Level Images, International Journal of Image and Graphics, Vol. 7, No. 4 (2007) 735-747.

[15] R. C. Gonzalez, R. E. Woods, 2004. "Digital Image Processing using MATLAB", Pearson Education, 2004,

[16] Velappa Ganapathy, and Kok Leong Liew, 2008, Handwritten Character Recognition Using Multiscale Neural Network Training Technique, Proceedings of World Academy of Science,Engineering and Technology Vol. 29 (May 2008) ISSN 1307-6884, 32-37. 